\title{
Robust Fault Detection for Networked Systems with Communication Delay and Data Missing *
}

\author{
Xiao He ${ }^{\mathrm{a}}$, Zidong Wang ${ }^{\mathrm{b}}$, D. H. Zhou ${ }^{\mathrm{a}}$ \\ ${ }^{\mathrm{a}}$ Department of Automation, TNList, Tsinghua University, Beijing 100084, P. R. China
}

${ }^{\mathrm{b}}$ Department of Information Systems and Computing, Brunel University, Uxbridge, Middlesex, UB8 3PH, United Kingdom

\begin{abstract}
In this paper, the robust fault detection problem is investigated for a class of discrete-time networked systems with unknown input and multiple state delays. A novel measurement model is utilized to represent both the random measurement delays and the stochastic data missing phenomenon, which are typically resulted from the limited capacity of the communication networks. Network status is assumed to vary in a Markovian fashion and its transition probability matrix is uncertain but reside in a known convex set of a polytopic type. The main purpose of this paper is to design a robust fault detection filter such that, for all unknown inputs, possible parameter uncertainties as well as incomplete measurements, the error between residual and fault is made as small as possible. By casting the addressed robust fault detection problem into an auxiliary robust $H_{\infty}$ filtering problem of a certain Markovian jumping system, a sufficient condition for the existence of the desired robust fault detection filter is established in terms of linear matrix inequalities. A numerical example is provided to illustrate the effectiveness and applicability of the proposed technique.
\end{abstract}

Key words: Fault detection; networked systems; parameter uncertainty; random measurement delay; data missing; Markovian jumping system.

\section{Introduction}

Control systems where sensors, controllers, actuators and other system components communicate over a communication network are a type of distributed control systems referred to as networked control systems (NCSs) [1,2]. The use of a communication network offers advantages in terms of reliability, enhanced resource utilization, reduced wiring and reconfigurability. As such, network-based analysis and designs have many industrial applications in, for example, automobiles, manufacturing plants, aircrafts, and HVAC systems. However, implementing a control network over a communication network induces stochastic delays and packet dropouts that inevitably degrade performance and could be a source of instability. The problem of designing NCSs against network-induced communication delays and packet dropouts has recently attracted considerable research attention, see $[3,4]$ for some representative works.

Fault detection and isolation (FDI), on the other hand, has been an active field of research over the past decades because of the ever increasing demand for higher performance, higher safety and reliability standards $[5,6]$. Generally speaking, a fault detection process consists of constructing a residual signal which can then be compared with a predefined threshold. When the residual exceeds the threshold, the fault is detected and an alarm is generated [7]. In view of the wide usage of the network ca-

\footnotetext{
* This work was supported by the NSFC under Grants $60721003 \& 60736026$, and national 973 project under grant 2009CB320602 \& 2010CB731800. Corresponding author D. H. Zhou.
}

Email address: zdh@tsinghua.edu.cn (D. H. Zhou). bles in today's world, a seemingly natural research problem is to study the FDI problems for networked systems in the presence of network-induced delays or data missing, see e.g. [8,9]. Since network-induced delays and data missing phenomenon are inherently random and timevarying [10], they have been modeled in various probabilistic ways [11-14]. Very recently, in [15], the networkinduced delay and data dropout problems have been investigated within a unified framework and the robust filtering problem with polytopic uncertainties has been thoroughly studied.

It should be pointed out that, in all the aforementioned results, it has been implicitly assumed that the delay or missing characteristics are statistically mutually independent from transfer to transfer. Obviously, such an assumption is quite restrictive since network-induced characteristics are highly related to each other over the time. One possible way to remove such an assumption is to describe the residual dynamics by a discrete-time Markovian jumping system (MJS) [16]. Unfortunately, exact transition probability matrix can not be obtained in practice and to the best of the authors' knowledge, the robust fault detection problem for networked MJSs with uncertain transition probability matrices has not been fully investigated, which constitutes the main focus of this paper.

In this paper, the robust fault detection problem is studied for a class of networked systems with unknown input, multiple state delays and data missing. A sequence varying in a Markovian fashion is employed in the measurement model, and both the measurement delays and data missing are simultaneously considered. Polytopictype uncertainty in the transition probability matrix of the Markov process is taken into account. The addressed robust fault detection problem is converted into an aux- 


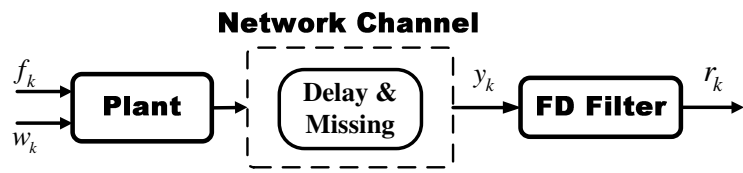

Fig. 1. Fault detection for networked systems

iliary robust $H_{\infty}$ filtering problem for a certain MJS, and a sufficient condition for the existence of the desired robust fault detection filters is established in terms of linear matrix inequalities (LMIs).

Notations used in the present paper are fairly standard except where otherwise stated. $\operatorname{Pr}\{e\}$ and $\mathbf{E}\{x\}$ represent, respectively, the occurrence probability of the event " $e$ " and the mathematical expectation of the stochastic variable $x . l_{2}[0, \infty)$ is the space of all square-summable vector functions over $[0, \infty)$, and $\|x\|$ is the standard $l_{2}$ norm of $x$, i.e., $\|x\|=\left(x^{T} x\right)^{1 / 2}$. In symmetric block matrices, "*" denotes the term that is induced by symmetry. $\operatorname{diag}\{\cdots\}$ stands for a block-diagonal matrix.

\section{Problem Formulation and Preliminaries}

Consider a class of discrete-time networked systems with the structure shown in Fig. 1. The plant and the robust fault detection filter are located at different places and connected with communication channel, which can be characterized by the following discrete-time linear system model:

$$
\left\{\begin{aligned}
x_{k+1} & =\sum_{i=0}^{q} A_{i} x_{k-i}+B_{w} w_{k}+B_{f} f_{k}, \\
y_{k} & =\sum_{i=0}^{q} \delta\left(\tau_{k}, i\right) C_{i} x_{k-i}+\bar{\delta}\left(\tau_{k},-1\right) D w_{k}, \\
x_{k} & =\varphi_{k}, \quad k=-q,-q+1, \ldots, 0,
\end{aligned}\right.
$$

where $x_{k} \in \mathbf{R}^{n}$ is the state vector; $w_{k} \in \mathbf{R}^{p}$ is the unknown input belonging to $l_{2}[0, \infty) ; f_{k} \in \mathbf{R}^{l}$ is the fault signal to be detected; $y_{k} \in \mathbf{R}^{m}$ is the measurement, which may contain both the random communication delays $(1 \leq i \leq q)$ and stochastic data missing $(q=-1)$ induced by the limited capacity of the communication networks. All system matrices in (1) are assumed to have appropriate dimensions and $\varphi_{k}$ is a given real initial sequence on $[-q, 0] . \bar{\delta}(j, l)=1-\delta(j, l)$, and $\delta(\cdot, \cdot)$ is the Kronecker delta function, i.e.,

$\delta(a, b)= \begin{cases}0, & \text { if } a \neq b \\ 1, & \text { if } a=b\end{cases}$

$\tau_{k}$ is a random variable introduced to describe the possibility of data missing as well as the size of the occurred delay at time instant $k$ [15]. In the present paper, we consider the sequence $\left\{\tau_{k}\right\}$ obeying a discrete-time homogeneous Markov chain taking values in the following finite state space

$\Xi=\{-1,0, \ldots, q\}$

and $\Lambda=\left[\lambda_{i j}\right]$ is the stationary transition probability matrix with its entities defined as

$\lambda_{i j}=\operatorname{Pr}\left\{\tau_{k+1}=j \mid \tau_{k}=i\right\}$.

In this paper, we deal with the robust fault detection problem for system (1) with polytopic uncertainties in the transition probability matrix $\Lambda$ of the Markov chain $\left\{\tau_{k}\right\}$ [17], namely $\Lambda \in \Pi$, where $\Pi$ is a polytope with $u$ vertices

$$
\Pi=\left\{\Lambda \mid \Lambda=\sum_{s=1}^{u} \beta_{s} \Lambda_{s} ; \quad \sum_{s=1}^{u} \beta_{s}=1, \quad \beta_{s} \geq 0\right\},
$$

where $\Lambda_{s}=\left[\lambda_{i j}^{(s)}\right](i, j \in \Xi, s=1, \ldots, u)$ are given transition probability matrices. It can be directly confirmed that the convex combination of these transition probability matrices is also a possible transition probability matrix.

Consider the following discrete-time full-order fault detection filter

$\left\{\begin{aligned} \tilde{x}_{k+1} & =G\left(\tau_{k}\right) \tilde{x}_{k}+K\left(\tau_{k}\right) y_{k}, \\ r_{k} & =L\left(\tau_{k}\right) \tilde{x}_{k}+M\left(\tau_{k}\right) y_{k},\end{aligned}\right.$

here, $\tilde{x}_{k} \in \mathbf{R}^{n}$ is the state of the fault detection filter; $r_{k} \in \mathbf{R}^{l}$ is its output (also called "residual") that is compatible with $f_{k}$. For each $\tau_{k}=i \in \Xi$, we notate matrices $G_{i}=G\left(\tau_{k}=i\right), K_{i}=K\left(\tau_{k}=i\right), L_{i}=L\left(\tau_{k}=\right.$ $i)$ and $M_{i}=M\left(\tau_{k}=i\right)$ as $G\left(\tau_{k}\right), K\left(\tau_{k}\right), L\left(\tau_{k}\right)$ and $M\left(\tau_{k}\right)$, respectively. In our present work, it is intended to make the error between the residual $r_{k}$ and the fault signal $f_{k}$ as small as possible in $H_{\infty}$ framework.

Introduce the following new vectors

$$
\begin{aligned}
\zeta_{k} & =\left[\begin{array}{ll}
w_{k}^{T} & f_{k}^{T}
\end{array}\right]^{T}, \quad \tilde{r}_{k}=r_{k}-\hat{f}_{k}, \\
\bar{x}_{k} & =\left[\begin{array}{lll}
x_{k-1}^{T} & \cdots & x_{k-q}^{T}
\end{array}\right]^{T}, \quad \eta_{k}=\left[\begin{array}{lll}
x_{k}^{T} & \bar{x}_{k}^{T} & \tilde{x}_{k}^{T}
\end{array}\right]^{T},
\end{aligned}
$$

and let matrices $\tilde{A}\left(\tau_{k}\right), \tilde{B}\left(\tau_{k}\right), \tilde{C}\left(\tau_{k}\right)$ and $\tilde{D}\left(\tau_{k}\right)$ represent $\tilde{A}_{i}=\tilde{A}\left(\tau_{k}=i\right), \tilde{B}_{i}=\tilde{B}\left(\tau_{k}=i\right), \tilde{C}_{i}=\tilde{C}\left(\tau_{k}=i\right)$ and $\tilde{D}_{i}=\tilde{D}\left(\tau_{k}=i\right)$. The overall fault detection dynamics governed by the following system can be obtained

$$
\left\{\begin{aligned}
\eta_{k+1} & =\tilde{A}_{i} \eta_{k}+\tilde{B}_{i} \zeta_{k}, \\
\tilde{r}_{k} & =\tilde{C}_{i} \eta_{k}+\tilde{D}_{i} \zeta_{k},
\end{aligned}\right.
$$

where

$$
\begin{aligned}
\tilde{A}_{i} & =\left[\begin{array}{ccc}
A_{0} & A_{d} & 0 \\
\hat{A}_{21} & \hat{A}_{22} & 0 \\
\delta(i, 0) K_{0} C_{0} & K_{i} C_{i} e_{i} & G_{i}
\end{array}\right], \\
\tilde{B}_{i} & =\left[\begin{array}{ll}
B_{w} & B_{f} \\
0_{q n \times p} & 0_{q n \times l} \\
\bar{\delta}(i,-1) K_{i} D & 0
\end{array}\right], \\
\hat{A}_{21} & =\left[\begin{array}{ll}
I_{n} \\
0_{(q-1) n \times n}
\end{array}\right], \hat{A}_{22}=\left[\begin{array}{ll}
0 & 0 \\
I_{(q-1) n} & 0
\end{array}\right], \\
A_{d} & =\left[\begin{array}{lll}
A_{1} & \cdots & A_{q}
\end{array}\right], \\
e_{i} & =\left[\begin{array}{lll}
\delta(i, 1) I_{n} & \cdots & \delta(i, q) I_{n}
\end{array}\right], \\
\tilde{C}_{i} & =\left[\begin{array}{lll}
\delta(i, 0) M_{0} C_{0} & M_{i} C_{i} e_{i} & L_{i}
\end{array}\right], \\
\tilde{D}_{i} & =\left[\begin{array}{ll}
\bar{\delta}(i,-1) M_{i} D & -I
\end{array}\right] .
\end{aligned}
$$

After the above treatments, the possible communication delays and data missing introduced by network cable can 
be converted as the jumping parameters of the Markovian jumping system (7) with the same transition probability matrix $\Lambda$ in original system (1).

Recall the following definition of mean square stability for MJSs.

Definition $1 \quad[18,19]$ System (7) with $\zeta_{k}=0$ is said to be mean square stable if

$\mathbf{E}\left\{\left\|\eta_{k}\right\|^{2}\right\} \rightarrow 0, \quad$ as $k \rightarrow \infty$

for any initial condition $\eta_{0}$ and initial distribution $\tau_{0} \in$ $\Xi$.

Considering the existence of uncertainty in the transition probability matrix of MJSs, we further introduce the following definition.

Definition 2 Markovian system (7) with uncertain transition probability matrix $\Lambda \in \Pi$ is robustly mean square stable if (7) is mean square stable for each $\Lambda \in \Pi$.

With Definition 2, the original robust fault detection filter design problem for system (1) can be further converted to a robust $H_{\infty}$ filtering problem [20] for an MJS (7): Finding a series of filter parameters $G_{i}, K_{i}, L_{i}$ and $M_{i}(i \in \Xi)$ such that the augmented fault detection dynamics $(7)$ is robustly mean square stable and the infimum of $\gamma$ is made as small as possible in the feasibility of

$$
\sup _{\zeta_{k} \neq 0} \mathbf{E}\left\{\left\|\tilde{r}_{k}\right\|^{2} /\left\|\zeta_{k}\right\|^{2}\right\}<\gamma^{2}, \quad \gamma>0
$$

Introduce a residual evaluation function $J(k)$ with a form of quadratic sum

$J(k)=\left\{\sum_{h=0}^{k} r_{h}^{T} r_{h}\right\}^{1 / 2}$,

and the occurrence of faults can then be alarmed by comparing the incremental version of $J(k)$ with a prescribed threshold $J_{t h}$, according to the following logic

$\left\{\begin{array}{l}J(k)-J(k-\mathcal{L})>J_{t h} \Longrightarrow \text { fault detected }, \\ J(k)-J(k-\mathcal{L}) \leq J_{t h} \Longrightarrow \text { no faults }\end{array}\right.$

where

$$
J_{t h}=\sup _{k \in \mathbf{N}+, w_{k} \in l_{2}, f_{k}=0} \mathbf{E}\{J(k+\mathcal{L})-J(k)\},
$$

and $\mathcal{L}$ is the length of a finite evaluating time horizon.

\section{Main Results}

In this section we shall discuss the robust fault detection filter design problem of system (1). The following Bounded Real Lemma (BRL) will help us in deriving the main result.

Lemma 3 [19] (Discrete BRL for MJSs) Consider the MJS (7) with fixed and known transition probability matrix (3). Let $\gamma>0$ be a given scalar. Then the system (7) is mean square stable with $\zeta_{k}=0$ and, under zero initial conditions, satisfies (9), if there exist matrices $\tilde{P}_{i} \in \mathbf{R}^{(q+2) n}$ such that the following LMIs

$$
\left[\begin{array}{cccc}
-P_{i} & \tilde{A}_{i}^{T} \mathcal{P}^{T} \mathcal{S}_{i} & 0 & \tilde{C}_{i}^{T} \\
* & -\mathcal{P}^{T} \mathcal{S}_{i} & \mathcal{P}^{T} \mathcal{S}_{i} \tilde{B}_{i} & 0 \\
* & * & -\gamma^{2} I & \tilde{D}_{i}^{T} \\
* & * & * & -I
\end{array}\right]<0
$$

hold for any $i \in \Xi$, where $\tilde{A}_{i}, \tilde{B}_{i}, \tilde{C}_{i}, \tilde{D}_{i}$ are defined in (8) and

$$
\begin{aligned}
\mathcal{P} & =\left[\begin{array}{lll}
P_{-1} & \cdots & P_{q}
\end{array}\right]^{T}, \\
\mathcal{S}_{i} & =\left[\begin{array}{lll}
\lambda_{i(-1)} I_{(q+2) n} & \cdots & \lambda_{i q} I_{(q+2) n}
\end{array}\right]^{T} .
\end{aligned}
$$

Now, we establish an alternative sufficient condition for MJS (7) with fixed and known transition probability matrix from Lemma 3. The proof is similar with that of Corollary 1 in [15] and is omitted here.

Lemma 4 LMIs (11) are feasible if there exist matrices $P_{i} \in \mathbf{R}^{(q+2) n}$ and $\mathcal{Q}_{i} \in \mathbf{R}^{(q+2) n}$ satisfying

$$
\left[\begin{array}{cccc}
-P_{i} & \tilde{A}_{i}^{T} \mathcal{Q}_{i}^{T} & 0 & \tilde{C}_{i}^{T} \\
* & \bar{\Gamma}_{22} & \mathcal{Q}_{i} \tilde{B}_{i} & 0 \\
* & * & -\gamma^{2} I & \tilde{D}_{i}^{T} \\
* & * & * & -I
\end{array}\right]<0,
$$

where $\bar{\Gamma}_{22}=\mathcal{P}^{T} \mathcal{S}_{i}-\mathcal{Q}_{i}-\mathcal{Q}_{i}^{T}$.

Next, we give the following sufficient $H_{\infty}$ filter analysis condition for MJS (7) with uncertain transition probability matrix $\Lambda \in \Pi$.

Lemma 5 Consider system (1) with uncertain transition probability matrix $\Lambda \in \Pi$. For a given fault detection filter of the form (5), the augmented dynamic (7) is robustly mean square stable and satisfies the constraint (9) if there exist matrices $P_{i s} \in \mathbf{R}^{(q+2) n}$, $H_{i} \in \mathbf{R}^{(q+2) n \times(q+2)^{2} n}, E_{i} \in \mathbf{R}^{(q+2)^{2} n \times(q+2)^{2} n}, Q_{i} \in$ $\mathbf{R}^{(q+2) n \times(q+2) n}$ such that the following $L M I s$

$$
\left[\begin{array}{ccccc}
-P_{i s} & \tilde{A}_{i}^{T} \mathcal{Q}_{i}^{T} & 0 & 0 & \tilde{C}_{i}^{T} \\
* & \Upsilon_{22} & \Upsilon_{23} & \mathcal{Q}_{i} \tilde{B}_{i} & 0 \\
* & * & \Upsilon_{33} & 0 & 0 \\
* & * & * & -\gamma^{2} I & \tilde{D}_{i}^{T} \\
* & * & * & * & -I
\end{array}\right]<0
$$

hold for all $i \in \Xi$ and $s=1, \ldots, u$, where $\tilde{A}_{i}, \tilde{B}_{i}, \tilde{C}_{i}, \tilde{D}_{i}$ are defined in (8) and

$$
\begin{aligned}
\Upsilon_{22} & =-\mathcal{Q}_{i}-\mathcal{Q}_{i}^{T}+H_{i} \mathcal{S}_{i s}+\mathcal{S}_{i s}^{T} H_{i}^{T}, \\
\Upsilon_{23} & =-0.5 \mathcal{P}_{s}^{T}+H_{i}+\mathcal{S}_{i s}^{T} E_{i}, \Upsilon_{33}=E_{i}+E_{i}^{T}, \\
\mathcal{P}_{s} & =\left[\begin{array}{lll}
P_{(-1) s} & \cdots & P_{q s}
\end{array}\right]^{T}, \\
\mathcal{S}_{i s} & =\left[\begin{array}{lll}
\lambda_{i(-1)}^{(s)} I_{(q+2) n} & \cdots & \lambda_{i q}^{(s)} I_{(q+2) n}
\end{array}\right]^{T} .
\end{aligned}
$$

Proof We firstly consider MJS (7) with an arbitrary fixed transition probability matrix. By taking into account (13) in Lemma 4, we further give the following sufficient condition ensuring that system (7) is mean square stable and satisfies the constraint (9): there exist matrices $P_{i}, \mathcal{Q}_{i}, H_{i}, E_{i}$ such that the following LMIs hold for all $i \in \Xi$. 


$$
\left[\begin{array}{ccccc}
-P_{i} & \tilde{A}_{i}^{T} \mathcal{Q}_{i}^{T} & 0 & 0 & \tilde{C}_{i}^{T} \\
* & \bar{\Upsilon}_{22} & \bar{\Upsilon}_{23} & \mathcal{Q}_{i} \tilde{B}_{i} & 0 \\
* & * & \Upsilon_{33} & 0 & 0 \\
* & * & * & -\gamma^{2} I & \tilde{D}_{i}^{T} \\
* & * & * & * & -I
\end{array}\right]<0
$$

where $\bar{\Upsilon}_{22}=-\mathcal{Q}_{i}-\mathcal{Q}_{i}^{T}+H_{i} \mathcal{S}_{i}+\mathcal{S}_{i}^{T} H_{i}^{T}, \bar{\Upsilon}_{23}=$ $-0.5 \mathcal{P}^{T}+H_{i}+\mathcal{S}_{i}^{T} E_{i} ; \Upsilon_{33}$ and $\mathcal{P}$ are the same as defined in (15) and (12), respectively. Pre- and post-multiply (16) by $\operatorname{diag}\left\{I, I,-\mathcal{S}_{i}^{T}, I, I\right\}$ and its transpose, and after some proper elementary transformation, we can easily get (13).

For an arbitrary uncertain system with transition probability matrix $\Lambda \in \Pi$, one can always find a set of coefficients $\beta_{s} \geq 0(s=1, \ldots, u)$ such that (4) holds. Noticing that LMIs (14) are affine in the matrices $P_{i s}$ and $\mathcal{S}_{i s}$, we multiply suitable inequalities of (14) by appropriate scalars $\beta_{s}$ and then summing up, it can be readily shown that (16) hold with matrices $P_{i}(\beta)=\sum_{s=1}^{u} \beta_{s} P_{i s}$. Thus, from (14), we can confirm that (13) holds for any choice of $\Lambda$. Form Lemma 4 and Lemma 3 , it follows that system (1) is robustly mean square stable and (9) is satisfied. This concludes the proof.

Next, we consider the robust fault detection filter design problem for system (1) with uncertain transition probability matrix $\Lambda \in \Pi$.

Theorem 6 Consider system (1) with uncertain transition probability matrix $\Lambda \in \Pi$ and let $\gamma>0$ be $a$ given scalar. Then there exists a full-order robust fault detection filter of the form (5) ensuring that the overall augmented dynamics (7) is robustly mean square stable and the constraint (9) is satisfied if, there exist matrices $0<X_{i s}^{T}=X_{i s} \in \mathbf{R}^{(q+2) n \times(q+2) n}, S_{i} \in \mathbf{R}^{n \times n}$, $Z_{i} \in \mathbf{R}^{n \times n}, Y_{i} \in \mathbf{R}^{n \times n}, \bar{G}_{i} \in \mathbf{R}^{n \times n}, \bar{K}_{i} \in \mathbf{R}^{n \times m}$, $\bar{L}_{i} \in \mathbf{R}^{l \times n}, \bar{M}_{i} \in \mathbf{R}^{l \times m}, N_{i} \in \mathbf{R}^{q n \times q n}, 0<\mathcal{H}_{i s}^{T}=\mathcal{H}_{i s} \in$ $\mathbf{R}^{(q+2) n \times(q+2) n}, \mathcal{E}_{i s} \in \mathbf{R}^{(q+2) n \times(q+2) n}$ such that the following LMIs

$$
\left[\begin{array}{ccccc}
-X_{i s} & \Psi_{12} & 0 & 0 & \Psi_{15} \\
* & \Psi_{22} & \Psi_{23} & \Psi_{24} & 0 \\
* & * & \Psi_{33} & 0 & 0 \\
* & * & * & -\gamma^{2} I & \tilde{D}_{i}^{T} \\
* & * & * & * & -I
\end{array}\right]<0
$$

hold for $i \in \Xi$ and $s=1, \ldots, u$, where

$$
\begin{aligned}
& \Psi_{12}=\left[\begin{array}{ccc}
A_{0}^{T} Z_{i}^{T} & \Psi_{12}^{(12)} & \Psi_{12}^{(13)} \\
A_{d}^{T} Z_{i}^{T} & \Psi_{12}^{(22)} & \Psi_{12}^{(23)} \\
A_{0}^{T} Z_{i}^{T} & \Psi_{12}^{(32)} & \Psi_{12}^{(33)}
\end{array}\right] \\
& \Psi_{15}=\left[\begin{array}{lll}
\delta(i, 0) \bar{M}_{0} C_{0}+\bar{L}_{i} & \bar{M}_{i} C_{i} e_{i} & \delta(i, 0) \bar{M}_{0} C_{0}
\end{array}\right]^{T}, \\
& \Psi_{22}=\mathcal{H}_{i s}+\mathcal{H}_{i s}^{T}-\left[\begin{array}{ccc}
\Psi_{22}^{(11)} & 0 & \Psi_{22}^{(13)} \\
* & \Psi_{22}^{(22)} & 0 \\
* & * & \Psi_{22}^{(33)}
\end{array}\right] \text {, } \\
& \Psi_{23}=-0.5 \mathcal{X}_{s} \mathcal{S}_{i s}+\mathcal{H}_{i s}+\mathcal{E}_{i s},
\end{aligned}
$$

$$
\begin{aligned}
& \Psi_{24}=\left[\begin{array}{cc}
Z_{i} B_{w} & Z_{i} B_{f} \\
0 & 0 \\
Y_{i} B_{w}+\bar{\delta}(i,-1) \bar{K}_{i} D & Y_{i} B_{f}
\end{array}\right], \\
& \Psi_{33}=\mathcal{E}_{i s}+\mathcal{E}_{i s}^{T}, \quad \mathcal{X}_{s}=\left[\begin{array}{lll}
X_{(-1) s} & \cdots & X_{q s}
\end{array}\right]^{T}, \\
& \Psi_{12}^{(12)}=\Psi_{12}^{(32)}=\left[\begin{array}{ll}
I_{n} & 0_{n \times(q-1) n}
\end{array}\right] N_{i}^{T}, \\
& \Psi_{12}^{(13)}=A_{0}^{T} Y_{i}^{T}+\delta(i, 0) C_{0}^{T} \bar{K}_{0}^{T}+\bar{G}_{i}^{T}, \\
& \Psi_{12}^{(22)}=\left[\begin{array}{ll}
0_{(q-1) n \times n} & I_{(q-1) n} \\
0_{n} & 0_{n \times(q-1) n}
\end{array}\right] N_{i}^{T}, \\
& \Psi_{12}^{(23)}=A_{d}^{T} Y_{i}^{T}+e_{i}^{T} C_{i}^{T} \bar{K}_{i}^{T}, \quad \Psi_{12}^{(33)}=A_{0}^{T} Y_{i}^{T}+\delta(i, 0) C_{0}^{T} \bar{K}_{0}^{T} \\
& \Psi_{22}^{(11)}=Z_{i}+Z_{i}^{T}, \quad \Psi_{22}^{(13)}=Z_{i}+Y_{i}^{T}+S_{i}^{T}, \\
& \Psi_{22}^{(22)}=N_{i}+N_{i}^{T}, \quad \Psi_{22}^{(33)}=Y_{i}+Y_{i}^{T} .
\end{aligned}
$$

$A_{d}, \tilde{D}_{i}$ are shown in (8); $\mathcal{S}_{i s}$ is the same in (15). Moreover, if (17) is feasible, the parameters of the desired robust fault detection filter can be given by

$G_{i}=V_{i}^{-1} \bar{G}_{i} S_{i}^{-1} V_{i}, \quad K_{i}=V_{i}^{-1} \bar{K}_{i}$,

$L_{i}=\bar{L}_{i} S_{i}^{-1} V_{i}, \quad M_{i}=\bar{M}_{i}$,

where $V_{i} \in \mathbf{R}^{n \times n}$ is any invertible matrix (for example, $V_{i}$ could be set as I).

Proof Consider the augmented parameters in (8), we take a special structure of $\mathcal{Q}_{i}$ into account. Let

$$
\mathcal{Q}_{i}^{T}=\left[Q_{i}^{a b}\right]_{3 \times 3},
$$

and introduce new matrices

$$
\overline{\mathcal{Q}}_{i}^{T}=\left[\bar{Q}_{i}^{a b}\right]_{3 \times 3}
$$

with their entities $Q_{i}^{11}=Y_{i}, Q_{i}^{13}=V_{i}, Q_{i}^{22}=X_{i}, Q_{i}^{12}=$ $Q_{i}^{21}=Q_{i}^{23}=Q_{i}^{32}=0, \bar{Q}_{i}^{11}=Z_{i}^{-1}, \bar{Q}_{i}^{13}=U_{i}, \bar{Q}_{i}^{22}=I$, $\bar{Q}_{i}^{12}=\bar{Q}_{i}^{21}=\bar{Q}_{i}^{23}=\bar{Q}_{i}^{32}=0$ and $Q_{i}^{31}, Q_{i}^{33}, \bar{Q}_{i}^{31}, \bar{Q}_{i}^{33}$ are uniquely determined from the following equalities

$$
\begin{aligned}
& {\left[\begin{array}{cc}
Y_{i} & V_{i} \\
Q_{i}^{31} & Q_{i}^{33}
\end{array}\right]\left[\begin{array}{cc}
Z_{i}^{-1} & U_{i} \\
\bar{Q}_{i}^{31} & \bar{Q}_{i}^{33}
\end{array}\right]=I} \\
& {\left[\begin{array}{cc}
Z_{i}^{-1} & U_{i} \\
\bar{Q}_{i}^{31} & \bar{Q}_{i}^{33}
\end{array}\right]\left[\begin{array}{cc}
Y_{i} & V_{i} \\
Q_{i}^{31} & Q_{i}^{33}
\end{array}\right]=I}
\end{aligned}
$$

Furthermore, we have the following relationship

$$
\mathcal{Q}_{i} \overline{\mathcal{Q}}_{i}=\overline{\mathcal{Q}}_{i} \mathcal{Q}_{i}=\operatorname{diag}\left\{I, N_{i}, I\right\} .
$$

Define

$$
\mathcal{T}_{i}=\left[T_{i}^{a b}\right]_{3 \times 3},
$$

with $T_{i}^{11}=Z_{i}^{T}, T_{i}^{13}=Y_{i}^{T}, T_{i}^{22}=I, T_{i}^{33}=V_{i}^{T}$ and other entities all zeros, performing congruence transformations to (14) by $\operatorname{diag}\left\{\overline{\mathcal{Q}}_{i}^{T} \mathcal{T}_{i}, \overline{\mathcal{Q}}_{i}^{T} \mathcal{T}_{i}, \mathcal{S}_{i s} \overline{\mathcal{Q}}_{i}^{T} \mathcal{T}_{i}, I, I\right\}$ and define

$$
\begin{aligned}
X_{i s} & =\mathcal{T}_{i}^{T} \overline{\mathcal{Q}}_{i} P_{i s} \overline{\mathcal{Q}}_{i}^{T} \mathcal{T}_{i}, \quad \mathcal{H}_{i s}=\mathcal{T}_{i}^{T} \overline{\mathcal{Q}}_{i} H_{i} \mathcal{S}_{i s} \overline{\mathcal{Q}}_{i}^{T} \mathcal{T}_{i}, \\
\mathcal{E}_{i s} & =\mathcal{T}_{i}^{T} \overline{\mathcal{Q}}_{i} \mathcal{S}_{i s}^{T} E_{i} \mathcal{S}_{i s} \overline{\mathcal{Q}}_{i}^{T} \mathcal{T}_{i}, \quad \bar{G}_{i}=V_{i} G_{i} U_{i}^{T} Z_{i}^{T}, \\
\bar{K}_{i} & =V_{i} K_{i}, \bar{L}_{i}=L_{i} U_{i}^{T} Z_{i}^{T}, \bar{M}_{i}=M_{i}, S_{i}=V_{i} U_{i}^{T} Z_{i}^{T},
\end{aligned}
$$

we can easily obtain that LMIs (17) with constraints (19) and (20) are sufficient condition for LMIs (14). Hence, if 
there exist matrices $X_{i s}>0, S_{i}, Z_{i}, Y_{i}, \bar{G}_{i}, \bar{K}_{i}, \bar{L}_{i}, \bar{M}_{i}$, $N_{i}, \mathcal{H}_{i s}>0$ and $\mathcal{E}_{i s}$ such that LMIs (17) are feasible, then the overall fault detection dynamics (7) is robustly mean square stable and the constraint (9) is satisfied.

Furthermore, from LMIs in (17), we can confirm that $\Psi_{22}<0$, which fuhrer indicates $\mathcal{H}_{i s}+\mathcal{H}_{i s}^{T}-\Psi_{22}>0$ and $Z_{i}$ and $Y_{i}$ are nonsingular. Define $\mathcal{W}=\left[\begin{array}{lll}I & 0 & -I\end{array}\right]$, we have

$\mathcal{W}\left[\mathcal{H}_{i s}+\mathcal{H}_{i s}^{T}-\Psi_{22}\right] \mathcal{W}^{T}=-S_{i}-S_{i}^{T}>0$,

which implies that $S_{i}$ is nonsingular and also ensures the existence of parameter matrices $G_{i}, K_{i}, L_{i}$ and $M_{i}$ in (18). The proof is completed.

Remark 7 In Theorem 6, an uncertainty-dependent robust fault detection filter design result is obtained, which is less conservative than the uncertainty-independent ones. In fact, if we impose

$X_{i s}=X_{i}, \quad \mathcal{H}_{i s}=\mathcal{H}_{i}, \quad \mathcal{E}_{i s}=\mathcal{E}_{i}$,

$i \in \Xi, \quad s=1, \ldots, u$,

to (17), the uncertainty-independent result is recovered.

Remark 8 In most practical cases in NCSs, we are able to know the size of the measurement delay or whether the data is missing at a certain time by using the time-stamp technique, and therefore the jumping parameters of the transformed MJS are accessible. In this sense, Theorem 6 provides us a network-status-dependent fault detection filter design result. On the other hand, if the network status is not accessible, i.e., the jumping parameters of the transformed MJS are unavailable, a network-statusindependent result can be easily obtained by imposing

$S_{i}=S, \quad V_{i}=V, \bar{G}_{i}=\bar{G}$,

$\bar{K}_{i}=\bar{K}, \quad \bar{L}_{i}=\bar{L}, \quad \bar{M}_{i}=\bar{M}, \quad i \in \Xi$,

to Theorem 6 .

Note that (17) is a set of LMIs over both the matrix variables and the prescribed scalar $\gamma^{2}$, which gives rise to the following two conclusions: (1) the robust full-order fault detection filter can be obtained from the solution of convex optimization problems in terms of LMIs that can be solved via efficient interior-point algorithms [21] and, (2) the scalar $\gamma^{2}$ can be also included as one of the optimization variables for LMIs (17), which makes it possible to obtain the minimal noise attenuation level bound for the fault detection dynamics (7). Thus, a suboptimal robust fault detection filter can be readily found by solving the following convex optimization problem.

Problem 1: Consider networked system (1) with multiple state-delays, unknown inputs and uncertain transition probability matrix $\Lambda \in \Pi$, a uncertainty-dependent sub-optimal robust fault detection filter can be obtained by solving the following problem:

$$
\begin{aligned}
& \min \\
& X_{i s}>0, S_{i}, Z_{i}, Y_{i}, N_{i}, \\
& \bar{G}_{i}, \bar{K}_{i}, \bar{L}_{i}, \bar{M}_{i}, \mathcal{H}_{i s}>0, \mathcal{E}_{i s} \\
& i \in \Xi, s=1, \ldots, u
\end{aligned}
$$

The parameters of the sub-optimal robust fault detection filter can be determined by (18), and the suboptimal robust $H_{\infty}$ attenuation level for fault detection dynamics is given by $\gamma^{*}=\sqrt{\gamma_{o p t}^{2}}$, where $\gamma_{o p t}^{2}$ are the sub-optimal solution of the corresponding convex optimization problem.

\section{A Numerical Example}

To illustrate the effectiveness of the proposed method, we provide a numerical example in this section. The parameters of the discrete-time networked system (1) are given as the follows:

$$
\begin{aligned}
& A_{0}=\left[\begin{array}{cc}
0 & 0.5 \\
0.2 & 0.2
\end{array}\right], A_{1}=\left[\begin{array}{cc}
0.2 & 0 \\
0.7 & 0.1
\end{array}\right], \quad B_{w}=\left[\begin{array}{c}
0.5 \\
0.3
\end{array}\right], \\
& B_{f}=\left[\begin{array}{c}
-1 \\
2
\end{array}\right], C_{0}=C_{1}=\left[\begin{array}{cc}
0.2 & 0 \\
0 & 0.5
\end{array}\right], \quad D=\left[\begin{array}{c}
0.2 \\
-0.1
\end{array}\right] .
\end{aligned}
$$

The initial state values $\varphi_{k}$ are set to be $\varphi_{-1}=\varphi_{0}=$ 0 . Letting $q=1$, the state-space of the Markov chain $\left\{\tau_{k}\right\}$ can be obtained as $\Xi=\{-1,0,1\}$. The transition probability matrix of the Markov process is unknown but resides in a polytope with the following two vertices:

$$
\Lambda_{1}=\left[\begin{array}{lll}
0.5 & 0.4 & 0.1 \\
0.3 & 0.4 & 0.3 \\
0.2 & 0.3 & 0.5
\end{array}\right], \Lambda_{2}=\left[\begin{array}{lll}
0.7 & 0.2 & 0.1 \\
0.3 & 0.4 & 0.3 \\
0.2 & 0.3 & 0.5
\end{array}\right] \text {, }
$$

The initial mode is set to be $\tau_{0}=0$. For $k=0,1, \ldots, 300$, the unknown input $w_{k}$ is supposed to be a random noise uniformly distributed over $[-0.5,0.5]$, and the fault signal $f_{k}$ is given as:

$$
f_{k}=\left\{\begin{array}{cl}
0.5, & \text { for } k=100,101, \ldots, 200 \\
0, & \text { others. }
\end{array}\right.
$$

With the above parameters, after solving Problem 1 by using the Matlab LMI toolbox [21], we can obtain the minimal noise attenuation level of the fault detection dynamic $\gamma_{o p t}=1.0000$. Furthermore, the parameters of the sub-optimal fault detection filter in different modes are given by

$$
\begin{aligned}
& G_{-1}=\left[\begin{array}{ll}
0.0025 & 1.2515 \\
0.0857 & 0.6038
\end{array}\right], K_{-1}=\left[\begin{array}{ll}
0 & 0 \\
0 & 0
\end{array}\right], \\
& L_{-1}=\left[\begin{array}{ll}
-0.6317 & 0.3461
\end{array}\right], M_{-1}=\left[\begin{array}{ll}
0 & 0
\end{array}\right], \\
& G_{0}=\left[\begin{array}{ll}
-0.1872 & 0.8228 \\
-0.0576 & 0.2541
\end{array}\right], K_{0}=10^{-6} \times\left[\begin{array}{ll}
-1.656 & -4.389 \\
-0.772 & -1.206
\end{array}\right], \\
& L_{0}=\left[\begin{array}{ll}
0.0734 & -0.3254
\end{array}\right], M_{0}=10^{-6} \times\left[\begin{array}{ll}
1.592 & -0.097
\end{array}\right], \\
& G_{1}=\left[\begin{array}{ll}
-0.3622 & 1.0477 \\
-0.2268 & 0.6846
\end{array}\right], K_{1}=10^{-6} \times\left[\begin{array}{ll}
-3.552 & -0.159 \\
-3.246 & -0.199
\end{array}\right], \\
& L_{1}=\left[\begin{array}{ll}
-0.3731 & 0.4892
\end{array}\right], M_{1}=10^{-6} \times\left[\begin{array}{ll}
1.534 & 0.159
\end{array}\right] .
\end{aligned}
$$

We consider the real transition probability matrix as

$\Lambda(\beta)=\left[\begin{array}{ccc}0.62 & 0.28 & 0.1 \\ 0.3 & 0.4 & 0.3 \\ 0.2 & 0.3 & 0.5\end{array}\right]$

which means $\beta_{1}=0.4, \beta_{2}=0.6$ in (4). For the sake of reducing false alarm as well as easy implementation, 


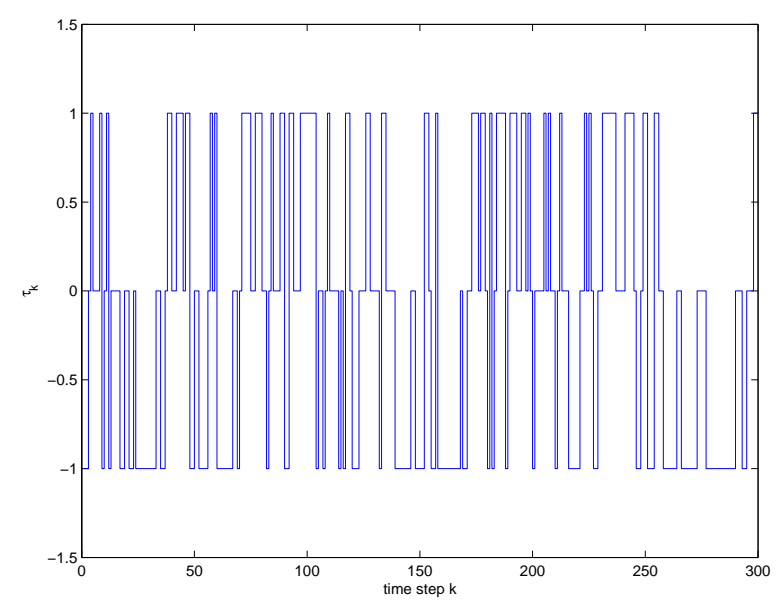

Fig. 2. Measurement mode over network

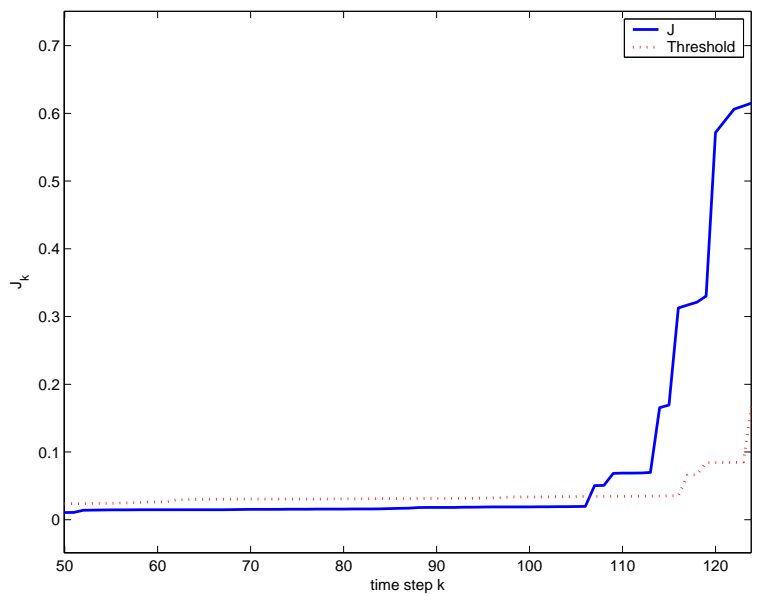

Fig. 3. Evolution of $J(k)$

we properly adjust the gain of the transfer function, enlarging the threshold be acceptable in practice. In this simulation, we multiple $K_{0}, M_{0}, K_{1}$ and $M_{1}$ with $10^{7}$. Using the resulted fault detection filter, we provide a time-domain simulation result.

Fig. 2 shows the measurement mode with random delay and missing phenomenon, where $\tau_{k}=-1$ (respectively, $0,1)$ means the measurement is missing (transmitted over the network perfectly, delayed for one-step, respectively).

The evolution function $J(k)$ defined in (10) is presented in Fig. 3. We set the length of the finite evaluating time horizon as $\mathcal{L}=10$ and determine a threshold by using 400 times Monte Carlo simulation as $J_{t h}=0.0156$. From Fig. 3, we can observe that when $k=107, J(k)>$ $J(k-\mathcal{L})+J_{t h}$ for the first time, and the designed fault detection filter can alarm the fault within 7 time steps after the fault occurred at $k=100$.

\section{References}

[1] L.A. Montestruque and P. Antsaklis, Stability of model-based networked control systems with time-varying transmission times, IEEE Transactions on Automatic Control, Vol. 49, No. 9, pp. 1562-1572, 2004.

[2] L. Schenato and B. Sinopoli and M. Franceschetti and K. Poolla and S.S. Sastry, Foundations of Control and Estimation Over Lossy Networks, Proceedings of IEEE, Vol. 49, No. 9, pp. 163-187, 2007.

[3] G.P. Liu and J.X. Mu and D. Rees and S.C. Chai, Design and stability analysis of networked control systems with random communication time delay using the modified MPC, International Journal of Control, Vol. 79, No. 4, pp. 288-297, 2006.

[4] J. Xiong and J. Lam, Stabilization of linear systems over networks with bounded packet loss, Automatica, Vol. 43, No. 1, pp. 80-87, 2007.

[5] P.M. Frank and S.X. Ding and B. Koppen-Seliger, Current developments in the theory of FDI, in: Proceedings of IFAC SAFEPROCESS, Budapest, Hungary, pp. 16-27, 2000.

[6] P. Zhang and S.X. Ding and G.Z. Wang and D.H. Zhou, Fault detection of linear discrete-time periodic systems, IEEE Transactions on Automatic Control, Vol. 50, No. 2, pp. 293$244,2005$.

[7] M. Zhong and S.X. Ding and J. Lam and H. Wang, An LMI approach to design robust fault detection filter for uncertain LTI systems, Automatica, Vol. 39, No. 3, pp. 543-550, 2003.

[8] H. Fang and H. Ye and M. Zhong, Fault diagnosis of networked control systems, in: Proceedings of IFAC SAFEPROCESS, Beijing, China, pp. 1-12, 2006.

[9] Z. Mao and B. Jiang and P. Shi, $H_{\infty}$ fault detection filter design for networked control systems modelled by discrete Markovian jump systems, IET Control Theory and Applications, Vol. 1, No. 5, pp. 1336-1343, 2007.

[10] D. Yue and Q. Han and J. Lam, Network-based robust $H_{\infty}$ control of systems with uncertainty, Automatica, Vol. 41 No. 6, pp. 999-1007, 2005.

[11] Z. Wang and D.W.C. Ho and X. Liu, Variance-constrained filtering for uncertain stochastic systems with missing measurements, IEEE Transactions on Automatic Control, Vol. 48, No. 7, pp. 1254-1258, 2003.

[12] Z. Wang and F. Yang and D.W.C. Ho and X. Liu, Robust $H_{\infty}$ control for networked systems with random packet losses, IEEE Transactions on Systems, Man, and Cybernetics - Part B, Vol. 37, No. 4, pp. 916-924, 2007.

[13] F. Yang and Z. Wang and Y.S. Hung and M. Gani, $H_{\infty}$ control for networked systems with random communication delays, IEEE Transactions on Automatic Control, Vol. 51, No. 3, pp. 511-518, 2006.

[14] L. Zhang and Y. Shi and T. Chen and B. Huang, A new method for stabilization of networked control systems with random delays, IEEE Transactions on Automatic Control, Vol. 50, No. 8, pp. 1177-1181, 2005.

[15] X. He and Z. Wang and D. Zhou, Robust $H_{\infty}$ filtering for networked systems with multiple state-delays, International Journal of Control, Vol. 80, No. 8, pp. 1217-1232, 2007.

[16] P. Zhang and S.X. Ding and P.M. Frank and M. Sader, Fault detection of networked control systems with missing measurements, in: Proceedings of Asian Control Conference, Melbourne, Australia, pp. 1258-1263, 2004.

[17] C.E. de Souza, Robust stability and stabilization of uncertain discrete-time Markovian jump linear systems, IEEE Transactions on Automatic Control, Vol. 51, No. 5, pp. 836-841, 2006.

[18] J. Lam and Z. Shu and S. Xu and E.K. Boukas, Robust $H_{\infty}$ control of descriptor discrete-time Markovian jump systems, International Journal of Control, Vol. 80, No. 3, pp. 374-385, 2007.

[19] J. Xiong and J. Lam, Stabilization of discrete-time Markovian jump linear systems via time-delayed controllers, Automatica, Vol. 42, No. 5, pp. 747-753, 2006.

[20] H. Gao and C. Wang, A delay-dependent approach to robust $H_{\infty}$ filtering for uncertain discrete-time state-delayed systems, IEEE Transactions on Signal Processing, Vol. 52, No. 6, pp. 1631-1640, 2004.

[21] S. Boyd, L. E. Ghaoui, E. Feron and V. Balakrishnan, Linear Matrix Inequalities in System and Control Theory, SIAM Studies in Applied Mathematics, Philadelphia, 1994. 